\title{
Correction to: Post-mortem tissue analyses in a patient with succinic semialdehyde dehydrogenase deficiency (SSADHD). I. Metabolomic outcomes
}

\author{
Trevor Kirby ${ }^{1} \cdot$ Dana C. Walters ${ }^{1} \cdot$ Madalyn Brown ${ }^{1} \cdot$ Erwin Jansen $^{2} \cdot$ Gajja S. Salomons $^{2} \cdot$ Coleman Turgeon $^{3}$. \\ Piero Rinaldo ${ }^{3}$. Erland Arning ${ }^{4} \cdot$ Paula Ashcraft $^{4} \cdot$ Teodoro Bottiglieri $^{4} \cdot$ Jean-Baptiste Roullet ${ }^{1} \cdot \mathrm{K}$ Michael Gibson $^{1}$
}

Published online: 18 April 2020

(C) Springer Science+Business Media, LLC, part of Springer Nature 2020

\section{Correction to: Metabolic Brain Disease} https://doi.org/10.1007/s11011-020-00550-1

Upon publication, it was noted that five of the on-line supplementary figures had incorrect figure: figure legend associations. These were supplementary Figs. 6, 7, 14, 15, and 23. The correct associations are as follows:

Suppl. Fig. 6: This figure refers to cerebral cortex $(\mathrm{CeC})$, not hippocampus.

Suppl. Fig. 7: This figure refers to liver, not cerebral cortex. Suppl. Fig. 14: The figure is incorrect, and the correct supplementary Fig. 14 corresponding to this legend is attached.

The online version of the original article can be found at https://doi.org/ 10.1007/s11011-020-00550-1

K. Michael Gibson

mike.gibson@wsu.edu

1 Department of Pharmacotherapy, Health Sciences Building Room, 210C, College of Pharmacy and Pharmaceutical Sciences, Washington State University, 412 E. Spokane Falls Boulevard, Spokane, WA 99202-2131, USA

2 Department of Clinical Chemistry, Metabolic Unit, Amsterdam, University Medical Center (Amsterdam UMC) and VU University, Medical Center (VUmc), Amsterdam, the Netherlands

3 Biochemical Genetics Laboratory, Department of Laboratory Medicine and Pathology, Mayo Clinic, Rochester, MN, USA

4 Baylor Scott \& White Research Institute, Institute of Metabolic Disease, Dallas, TX, USA

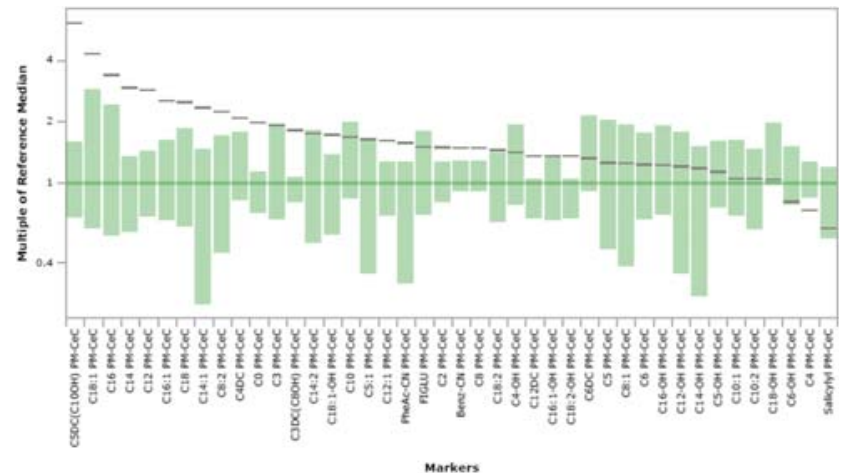

Suppl. Fig. 15: The figure is incorrect, and the correct supplementary Fig. 15 corresponding to this legend is attached.

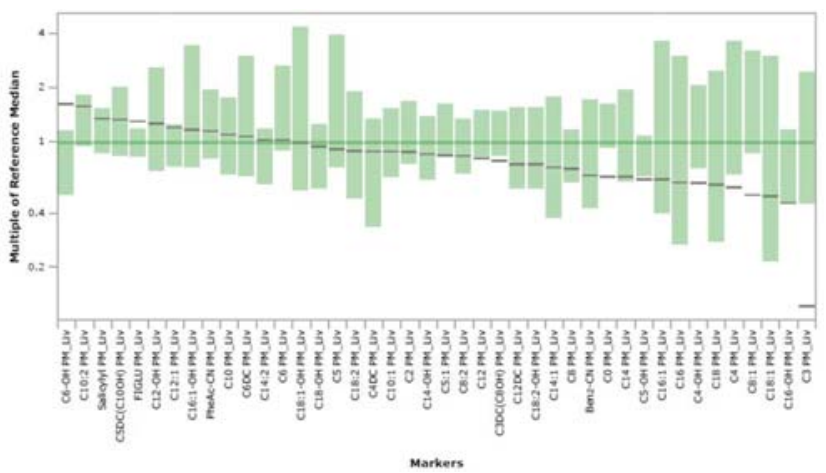

Suppl. Fig. 23: The figure legend is incorrect. The appropriate legend is as follows:

Suppl. Fig. 23. Quantitation of GABA-related metabolites in liver and kidney using isotope dilution mass spectrometry. Data is depicted as range (mean \pm SEM) for control $(n=4)$ in both liver (liv) and kidney (kid), as well as showing the individual patient value. Abbreviations: P, 
patient; C, control; Liv, liver; Kid, kidney; total GABA (including both free and esterified GABA), $\gamma$-aminobutyric acid; GHB, $\gamma$-hydroxybutyric acid; 4,5-DHHA, 4,5dihydroxyhexanoic acid; 4-GBA, 4-guanidinobutyric acid; SSA, succinic semialdehyde; GUAC, guanidinoacetic acid.
Since only a single value for the patient was obtained, statistical evaluation was not undertaken.

Publisher's note Springer Nature remains neutral with regard to jurisdictional claims in published maps and institutional affiliations. 